\title{
On the Translator's Subjectivity in the Conversion of Untranslatability of Classical Chinese Poetry
}

\author{
Yan Li \\ Department of Foreign Languages \\ The Engineering \& Technical College of Chengdu University of Technology \\ Leshan, Sichuan, China \\ 295199077@qq.com
}

Keywords: Untranslatability; Poetry; Translator's Subjectivity

\begin{abstract}
Poetry is regarded as the most difficult text to be translated and considered untranslatable to some extent. Thus, translators' subjectivity is a very important factor in the process of translating, converting the untranslatability into translatability. This paper is about to discuss how a translator deals with the untranslatability of classical Chinese poetry when it is translated into English, in terms of language features, forms and content.
\end{abstract}

\section{Introduction}

Poetry is one of the literary categories parallel to fiction, prose, drama and literature of movies and television, and also is the most concentrated literary style reflecting the social life with rich imagination and emotion expressed in a direct way, whose language is different from that of prose in the extent of conciseness and harmoniousness and especially the distinct rhythm [1]. Therefore, concerning its language features, form and content, to some translators, poetry is very difficult to be translated or untranslatable because the target language and source language have differences in nature of the two to some extents, and would lose some flavors during the translation even when a poem is translated into the target language. Despite the difficulties in poetry translation, a lot of excellent translated works have been produced. Therefore, translators' subjectivity plays a key role in the translation

. Then, this paper is to probe into the importance of the translators' subjectivity in the process of poetry translation with the examples of translated works of classical Chinese poems to prove that the so-called untranslatable really can be translated.

\section{Untranslatability of Classical Chinese Poetry}

What is untranslatability? It is inappropriate to define untranslatability as a fixed concept. Venuti Lawrence holds that linguistic culture can be divided into five parts: ecology, material culture, social culture, religious culture, and linguistics culture [2]. So it goes without saying that there are some absolute cultural differences between original language and target language, and certain information loss caused by translation is normal and objective. Therefore, cultural translatability is relative, whereas untranslatability is absolute. However, more translators maintain that there is no necessity to define untranslatability as an absolute conception, because translatability has its own limitations, so is untranslatability. Therefore, this section is to discuss the causes for the untranslatable of classical Chinese poetry-its language features, form and content.

Language Features. It is known that Chinese is a tone and monosyllabic language, and the most important feature of Chinese is that it is written as characters instead of alphabet which is the ultimate source of many of characteristics of Chinese poetry. Thus, the meaning conveyed by such characters is determined by the four tones - level, rise, fall-rise, and fall [3]. And a Chinese word, as in English, does not always have one clear-cut, fixed meaning, but often covers different meanings and even word classes and possesses rich implications, which makes possible the expression of thought and 
emotion with greatest economy of words. Besides, association is very important in Chinese, which includes notional associations, auditory associations and contextual associations [1]. Notional associations are aroused not by the sound, but by the visual form of the characters, such as “日”(sun), “月”(moon), “柳”(willow), etc.. Auditory associations are aroused by the sound of the character. And all conscious and unconscious puns are based on them. For instance, in the line “道是无晴却有晴” written by Liu Yuxi, the poet puns on the character “晴” (sunny) which has the same sound as the character “情” (love) [1]. And contextual associations are connected in the reader's mind because of some familiar literary context. For example, Chinese readers will associate “雪花”(snow) with “芦 花”(reed catkins), as occur in the poem “咏雪” ( snow ) “千片万片无数片, 飞入芦花总不见”.

Compared with the English sentence structures, Chinese sentence structures are shorter and less rigid without strict grammatical requirements, for Chinese does not have as many inflections, derivations, conjunctions, prepositions as English does. And for a Chinese sentence structure, the subject and the predicate are shared by various components, and the subject could disappear if it can be implied by the context, which is different from English, as the subject should be shared by the nominal components, while the core of the predicate by the verb in an English sentence. For instance, “天高云淡”(the sky is high and the clouds are pale). In this sentence, the predicates of the Chinese sentence are the adjectives “高”(high) and “淡”(pale).

Form. There are strict rules for the forms of classical Chinese poetry, which mainly are represented by the tones of the characters in the lines of the poems. Usually, the four tones are classified as two types in classical Chinese poems, the level tones (including level and rise), and oblique tones (fall-rise and fall), such as in the poem “春眠(level)不觉(level)晓，处处(oblique)闻啼 (oblique)鸟.夜来(level)风雨(oblique)声，花落(oblique)知多(level)少?”. For each line of a poem, the characters in even numbers should be given the tones as required, while the characters in odd numbers need not. In the writing of the classical Chinese poems, the rhythm is realized by the level and oblique tones, and the level and oblique tones should be parallel in the poem lines. And the same time, each line would express a thing on the whole and even lines of a poem must end with characters which have the same or similar sound, such as in the poem “红豆生南国，春来发几枝[i])? 愿君多采撷，此 物最相思[i]。” In general, if there are four lines in a poem, then the second and fourth lines should end with the characters which share the similar sound, as illustrated in the above example.

However, the rhythm of the English poems is realized by the stress and classified as iambic, trochee, anapest, dactyl, spondee, pyrrhic, and amphibrach [3]. And for an English poem, a line or more than one line would express a thing as a whole, and there are mainly three types of rhyme, end rhyme, such as "And answer, 'Let one living head, /But one arise,--we come, welcome![ $\wedge \mathrm{m}] /$ This but the living who are dumb[^m]')" [3], alliteration such as "Life is the lust of a lamp for the light that is dark till the dawn of the day that we die.", and assonance, such as "They lowing herd wind slowly, o'er the lea..." [3].

Content. Poetry is an inseparable unit of form and content. Then as there are many differences in Chinese and English cultures, the contents of the poems of the two are expressed differently to some extent, and the terms, especially associated with the culture and history, influence the understandings and translations of the poems in the two languages. For example, in the poem “名成八阵图, 功盖三 分国” written by Du Fu, “八阵图” and “三分国” refer respectively to a military strategy and the Three Kingdoms (220-265)—Wei, Shu Han and Wu in Chinese history. Besides, as Chinese tend to connect all things as a whole and describe things in a vivid way, it is known that there are vivid picture in the classic Chinese poetry, but also it is fuzzy in the descriptions. For example, in the poem “千山鸟飞绝，万径人踪灭”written by Liu Zongyuan, “千山” does not really mean “a thousand mountains" and “万径" “ten thousand paths". The poet just tries to use the two numbers to show that all mountains and paths. Take another example, “南朝四百八十寺, 多少楼台烟雨中” written by Du $\mathrm{Mu}$. The numbers in the two lines above are not really exact numbers of the temples or buildings. In order to describe the beautiful spring in the south of China, the poet takes advantage of these fuzzy words and gives us very beautiful scenery to appreciate. 


\section{Translator's Subjectivity}

With respect to the definition of the translator's subjectivity, some scholars and translators have attempted to provide numerous descriptions. Yu Guoyuan and Zhu Xianlong term the translator's subjectivity as "the initiative the translator displays under constraints other translation participants, external forces and his/her horizons in the process of translating" [4]. And ZhaMingjian and Tian Yu holds that the translator's subjectivity is the initiative the translator undertakes in the process of translating on the premise that he respects the source text [5]. However, the definition given by GaoNing is that the translator's subjectivity is reflected from two aspects: one is the subjectivity in interpreting the source text, and the other is the subjectivity in the formation of the target text [7]. From the above definitions, it can be inferred that the translator's subjectivity is neither arbitrary nor unrestricted; the translator can never exercise his or her own initiative without the constraint from inside and outside of the translator, namely, the subjectivity should neither be exaggerated nor rejected. Therefore, a rough definition of the translator's subjectivity is put forward: translator's subjectivity is the initiative and creativity that the translator manifests in translation.

\section{The Conversion of Untranslatability of Chinese Poetry}

As is discussed above, classical Chinese poetry is marked by strict tonal patterns and rhyme schemes while English poetry may be determined by the stress of the words in the lines as well as by conventional metrical patterns or rhyme schemes. Then in the process of translation, translators have tried to find a better way to solve these problems, for they believe they could find an effective one, just as Bassnett claims that the translator can at times enrich or clarify the source target text as a direct result of the translation process with his own initiative and creativity--his subjectivity, which would be illustrated in the following two examples [7].

“忆江南” is written by BaiJuyi in Tang Dynasty, which describes the very beautiful scenery of the certain place in the southern part of China, which appears in many poems to express the poets' complex feelings towards that place. The whole poem is “江南好, 风景旧曾谙.日出江花红胜火, 春 来江水绿如蓝. 能不忆江南?” Here are two translated versions of the poem: one is translated by Gong Jinghao as "Yi Jiang Nan/ Jiangnan is an alluring land./ Oh, the sights and sounds I knew so well;/ At sunrise the flowers on river banks/ Were suffused with a flame-like red./ In springtime, the river water turned turquoise blue. /How can I help remembering? " [2]; and another is translated by Chen Gang as"Fair South Recalled/ Fair southern shore / With scenes I much adore/ As sunrise riverside flowers more red than fire,/ In spring green river waves grow as blue as sapphire,/ Which I can't but admire." [2]. In the two versions, the two translators have interpreted the source text from different perspectives. Gong pays more attention to the content and language of the source than the form. Then in his translated version, we can appreciate the profound Chinese culture and the very essence of the emotion of BaiJuyi. In order to meet the needs of rhyme scheme and get the meanings, he translates “风景” as “the sights and sounds”, and “红胜火” as “flame-like red” and “绿如蓝” as "turquoise-blue". Chen's version is more on the form, and tries to display the Chinese culture in an overall sense. In his version, the sentence structures imitate the source text and considering the writing of English poems, he changed the end rhyme of the source text ABCBB into AABBB.

Another poem “春晓” is written by MengHaoran. The whole poem goes like this, “春眠不觉晓， 处处闻啼鸟. 夜来风雨声，花落知多少?” For this famous poem, there are lots of English translations. Here are two versions which are well accepted by people. The first is translated by XuYanchong in 1998 which is "A Spring Morning/ This morn of spring in bed I'm lying, / Not woke up till I hear birds crying. / After one night of wind and showers, / How many are the fallen flowers!" [8]. The second was translated by WengXianliang in 1983, which is "One Morning in Spring/ Late! This spring morning as I awake I know./ All around me the birds are crying, crying, / The storm last night, I sensed its fury. How many, / I wonder, are faller, poor dear flowers!" [8]. In the two versions, both translators give us the detailed descriptions about the birds' sound, flowers and rain, express the sadness to spring and avoid the fuzzy words “多少” by translating it into "how many". But when they 
translate these images, they have their own understandings and translation strategies to tackle. It is clear that Xu's takes account of form and content, while Weng's mainly focuses on the content and subtle emotions in the source text. Xu tries to translate the source text according to the English writing habit and rhyme scheme, and then in his translation, the first end rhymes are changed into AABB, and for lines, the rhythm is very similar to each other according to the stress. But Weng's does not consider the form of the target text. Instead, it pays more attention to the content. For example, he translates “春眠不觉晓” as “Late! This spring morning as I awake I know” describes the poet's regret about getting up late expressively.

It is noted that translators' subjectivity is very important in the translation process, for the experiences, values and levels of language of the translators will greatly affect their translated works.

\section{Conclusion}

Poetry, for translators, is regarded as untranslatable, for they think poetry is the inseparable unit of emotion and image, and the unit cannot be translated without any loss. Even Nida, under his functional equivalence theory, also thinks that there exists to some extent incommensurability between Chinese and English, and when a poem is being translated, translators focus more on its form than on its content, for the content is closely linked with its form. But with translator's initiative and creativity, the untranslatable can be converted in the translation.

\section{References}

[1] Peng Xingwang, "On the Characteristics of Chinese and English Poetry and Loss and Gain in Poetry Translation," Shanxi University, 2007.

[2] He Jun, "Converting Untranslatability of Poetry into Translatability through Translator's Recreating,' Journal of Chengdu University, vol. 4, 2008, pp. 124-127.

[3] Wang Jia, "From the Phonological Analysis of English and Chinese Poetry to See the Translatability and Untranslatability of English and Chinese Poetry," Journal of Nanchang College of Education, vol. 27, 2012, pp. 158-159.

[4] Yu Guoyuan and Zhu Xianlong, "Yizhe Zhutixing: Chanshixing de Chanshi," Foreign Languages Department of Central South University, vol. 6, 2003, pp. 8-14.

[5] Zha Mingjian and Tian Yu, "On the Subjectivity of the Translator," Chinese Translators Journal, vol, 2003, pp. 19-24.

[7] Gao Ning, "Lun Yizhe Zhutixing Diwei-Jian Lun Fanyi Biaozhun de Sheli Yuanze," Shanghai Journal of Translators, vol. 1, 1997, pp. 6-9.

[8] Xie Shijian and Wang Yanfen, "Translators' Subjectivity and Creativity in Poetry Translaton: A Case Study of Five Versions of 'Chunxiao'," Journal of Guilin Normal College, vol. 29, 2015, pp. 80-83. 